\title{
Fast Nonlinear Susceptibility Inversion with Variational Regularization
}

Carlos Milovic $^{1,2}$, Berkin Bilgic ${ }^{3}$, Bo Zhao ${ }^{3}$, Julio Acosta-Cabronero ${ }^{4}$, and Cristian Tejos ${ }^{1,2}$

1 Department of Electrical Engineering, Pontificia Universidad Catolica de Chile, Santiago, Chile

2 Biomedical Imaging Center, Pontificia Universidad Catolica de Chile, Santiago, Chile

3 Martinos Center for Biomedical Imaging, Harvard Medical School, MA, USA

4 Wellcome Centre for Human Neuroimaging, Institute of Neurology, University College London, London, UK

Corresponding author: Carlos Milovic, cmilovic@uc.cl

Manuscript typo: Full paper

Word count: 2796

Running title: FANSI-QSM

Abstract:

Purpose: Quantitative susceptibility mapping (QSM) can be performed through the minimization of a functional consisting of data fidelity and regularization terms. For data consistency, a Gaussian phase-noise distribution is often assumed, which breaks down when SNR is low. A previously proposed alternative is to use a nonlinear data fidelity term, which reduces streaking artifacts, mitigates noise amplification and results in more accurate susceptibility estimates. We hereby present a novel algorithm that solves the nonlinear functional while achieving computation speeds comparable to those for a linear formulation.

Methods: We developed a nonlinear QSM algorithm (FAst Nonlinear Susceptibility Inversion, FANSI) based on the variable splitting and alternating direction method of multipliers (ADMM), where the problem is split into simpler sub-problems with closed-form solutions and a decoupled nonlinear inversion hereby solved with a Newton-Raphson iterative procedure. FANSI performance was assessed using numerical phantom and in vivo experiments, and was compared against the nonlinear morphology-enabled dipole inversion (NMEDI) method.

Results: FANSI achieves similar accuracy to NMEDI but with significantly improved computational efficiency.

Conclusion: The proposed method enables accurate reconstructions in a fraction of the time required by state-of-the-art QSM methods.

Keywords: nonlinear inversion, quantitative susceptibility mapping, total variation, augmented Lagrangian, 


\section{Introduction}

Quantitative susceptibility mapping (QSM) involves estimating tissue susceptibilities from the phase of a gradient-recalled echo (GRE) acquisition. The GRE signal phase is proportional to the local reaction field emanating from magnetic susceptibility differences between tissues. However, solving the field-to-source (i.e. inverse) QSM problem is often challenging because the dipole kernel approximation has zero-valued coefficients across a double-conical surface in kspace, the so-called "magic cone” $(1,2)$. The direct dipole kernel inversion, thus, leads to divisions by zero for such frequency coefficients, which contaminates the reconstruction with streaking artifacts. Truncated approximations of the inverse dipole kernel can reduce but not eliminate streaking (3), and are prone to noise amplification (4). Consequently, in an effort to improve reconstruction quality, susceptibility inversions have been reformulated as an optimization problem usually comprising two terms: one ensuring data fidelity and a regularizer. From a Bayesian standpoint, the data fidelity term minimizes the discrepancy between a forward model and the MRI measurement while accounting for a given phase-noise distribution. The regularization term, conversely, constrains the impact of the zero-valued kernel coefficients by enforcing some level of prior knowledge about the solution. Typically, regularization promotes smoothness or sparsity in some domain, e.g. for the solution's first derivative (5-11), which leads to improved artifact and noise-amplification control (12). The total generalized variation (TGV) penalty, for example, promotes piece-wise smoothness $(13,14)$, hence mitigating the often piecewise patchy appearance resulting from total variation (TV) regularization (15-17).

Only a few studies, however, have focused on improving the formulation of the data fidelity term - a key aspect to improve QSM. In conventional approaches, data fidelity consists of a linear susceptibility-to-field relationship that assumes Gaussian noise; however, the phase-noise distribution deviates from a Gaussian density function for low signal-to-noise ratio (SNR) (18). In order to address this shortcoming, Liu et al. (19) proposed a nonlinear data fidelity formulation in which the measurement noise is modeled as a complex-valued Gaussian. A subsequent study demonstrated that this results in reduced streaking artifacts, noise mitigation, and hence, overall improvements in reconstruction accuracy (12). While effective, the previously proposed nonlinear morphology-enabled dipole inversion (NMEDI) algorithm (19) is computationally slow, obstructing online image calculation on standard vendor software, and making automated parameter selection (e.g. via L-curve analysis) unfeasible for the clinical 
routine. In this paper, we present a novel fast QSM algorithm with nonlinear consistency and variational regularization using variable splitting (20) and the alternating direction of multipliers method (ADMM) (17,20-23). We compared the accuracy and computational efficiency of this new algorithm against NMEDI, and demonstrated more than an order of magnitude speed-up with comparable reconstruction results.

\section{Theory}

Previously, Liu et al. (19) proposed the following optimization problem for nonlinear QSM inversion:

$$
\operatorname{argmin}_{x} \frac{1}{2}\left\|W\left(e^{i F^{H} D F x}-e^{i \phi}\right)\right\|_{2}^{2}+R(X)
$$

where $D$ is the magnetic dipole kernel in the frequency domain, $\quad \chi$ is the susceptibility distribution, $\phi$ is the tissue phase and $F$ is the Fourier operator with inverse, $F^{H} . W$ denotes a spatially-variable weight estimated from the normalized magnitude image, and $R(X)$ is the regularization term. NMEDI is an iterative reconstruction approach consisting of two nested loops. In the inner loop, NMEDI applies a Taylor expansion around the current susceptibility estimate and solves the ensuing linear system via conjugate gradients. In the outer loop, it introduces a quasi-Newton fixed-point solver to help improve the convergence.

ADMM solvers, however, constitute a faster alternative with potential for higher convergence rates via fewer iterations (17). In this work, we extend the ADMM framework to solve the nonlinear QSM inversion problem with a novel variable-splitting scheme. To this end, we introduced an auxiliary variable, $z=F^{H} D F X$, and decoupled the equation system, leading to the following augmented Lagrangian functional:

$$
\operatorname{argmin}_{X, z} \frac{1}{2}\left\|W\left(e^{i z}-e^{i \phi}\right)\right\|_{2}^{2}+\frac{\mu}{2}\left\|F^{H} D F X-z+s\right\|_{2}^{2}+R(X)
$$

where $s$ is the Lagrangian multiplier (a relaxation term), and $\mu$ is the penalty parameter. With the proposed variable splitting strategy, the optimization involving regularization and data fidelity terms can be treated as two separate sub-problems. The sub-problem for $X$ can be written as follows: 


$$
\operatorname{argmin}_{\chi} \frac{\mu}{2}\left\|F^{H} D F \chi-z+s\right\|_{2}^{2}+R(\chi),
$$

Eq. 3.

which has a closed-form solution as previously shown in (17).

The sub-problem for $z$ can be written as:

$$
\operatorname{argmin}_{z} \frac{1}{2}\left\|W\left(e^{i z}-e^{i \phi}\right)\right\|_{2}^{2}+\frac{\mu}{2}\left\|F^{H} D F \chi-z+s\right\|_{2}^{2} \quad \text { Eq. } 4 .
$$

Applying the Euler formula, the above equation can be re-written as:

$$
\operatorname{argmin}_{z}-\sum\left[W^{2} \cos (z-\phi)\right]+\frac{\mu}{2}\left\|F^{H} D F \chi-z+s\right\|_{2}^{2} \quad \text { Eq. } 5 .
$$

In such form, the gradient of the functional can be expressed as a voxel-wise decoupled structure:

$$
\partial f_{z}=W^{2} \sin (z-\phi)+\mu z-\mu\left(F^{H} D F \chi+s\right),
$$

Eq. 6.

for which a first order approximation $(z \approx \phi)$ yields:

$$
z=\frac{W^{2} \phi+\mu\left(F^{H} D F X+s\right)}{W^{2}+\mu}
$$

The above equation provides a solution that is consistent with a spatially-weighted linear problem. Eq. 7 may be applicable to a wide range of SNR regimes, with the caveat that it is markedly vulnerable to phase-wrap errors.

A more robust solution was found solving for the roots of the nonlinear function using a NewtonRaphson (NR) voxel-wise approach (24).

$$
z_{n}=z_{n-1}-\frac{W^{2} \sin (z-\phi)+\mu z_{n-1}-\mu\left(F^{H} D F \chi+s\right)}{W^{2} \cos (z-\phi)+\mu}
$$

NR solvers are fast (converge quadratically) but are highly dependent on initial conditions, i.e. they might return local minima or become unstable when the denominator approaches zero. In order to stabilize the solver, we propose using the minimum of the consistency term between $X$ and $z$ as the starting point, i.e.:

$$
z_{0}=F^{H} D F X+s
$$

Eq. 9. 
As such, the consistency between $z$ and $\phi$ is considered as a perturbation term. If $W \leq 1$, and $\mu \geq 1$, then no other local minima exist, and the global minimum is within $\pi$ relative to this point.

Finally, we need to update $s$ :

$$
s=s+z-F^{H} D F X \quad \text { Eq. } 10 .
$$

We iterate the solutions for each subproblem ( $x$ and $z$ ) until convergence.

The source code for the proposed reconstruction is publicly available as part of the FANSI (Fast Nonlinear Susceptibility Inversion) toolbox http://gitlab.com/cmilovic/FANSI-toolbox.git.

\section{Methods}

The proposed nonlinear solver was compared both with NMEDI (19) and with a simpler (linear data fidelity) ADMM (17) formulation (Eq. 7 using $W=1$ ). We assessed both TV and TGV (17) penalties with the ADMM solver, with results for TV regularization shown in the main manuscript and for TGV as supplementary material (Figs S7, S8, S9, S10, S11 and S12, and Table S1). It should be highlighted that NMEDI's regularizing penalty consists of a spatiallyweighted TV norm that incorporates magnitude-edge information. In the forthcoming sections, linear-fidelity solutions with ADMM will be referred to as “TV” and nonlinear solutions as "TVFANSI”.

The following experiments were carried out to evaluate the performance of the proposed method:

Synthetic-brain phantom (25): This experiment aimed at evaluating algorithm responses to different sources of error and SNR dependencies. Base susceptibility values were used to synthesize the magnetic field map, which in turn was expressed as a signal-phase distribution. Magnitudes, normalized to the [0,1] range, were set proportional to susceptibility values. Real and imaginary parts of the simulated signal were corrupted independently with Gaussian noise (SD: 1/345), and new magnitude and phase data were calculated. Such procedure generated an SNR gradient in the simulated thalamus. Additionally, four spheres were added (13 voxels in diameter) to simulate different lesions (Fig. S2). Each lesion was of constant susceptibility (-0.5, -0.3, 0.6 and $1.2 \mathrm{ppm}$, respectively) and zero magnitude to simulate an extreme $\mathrm{T}_{2} *$ dephasing regime (e.g. using excessively long TE). Residual errors (e.g. from imperfect unwrapping, coil or 
multi-echo combinations) were also simulated by degrading the resulting phase with the introduction of five spatially-separate $2 \pi$ jumps (positive or negative). Global (and local withinsphere) RMSE scores were used as accuracy measures to evaluate algorithm performance. It should be noted that this phantom is intrinsically piece-wise constant, thus we only provide RMSE scores for the new approach in contrast to NMEDI without gradient-weighting, since gradient-weighting constitutes a major advantage in this particular scenario. Local RMSE scores were normalized by the mean ground-truth susceptibility for each sphere.

COSMOS-brain (26) forward simulation: A COSMOS reconstruction (27) - from fast 3D gradient-echo scans, 15-fold acceleration, acquired at twelve different head orientations on a 3T Siemens Trio scanner (Siemens, Erlangen, Germany) with a 32-channel head-coil, $1.06 \times 1.06 \times 1.06-\mathrm{mm}^{3}$ isotropic voxels, $240 \times 196 \times 120$ matrix size, flip angle $=15^{\circ}$ and TE/TR=25/35ms - was used as a susceptibility ground truth from which a local field map was synthesized. The magnitude from a single acquisition, normalized to unity, was used to simulate complex image data, which was then corrupted by complex-valued Gaussian noise with 2\% amplitude (peak-SNR=50). Resulting susceptibility distributions were compared with the COSMOS ground-truth using RMSE scores (low value=high consistency), high-frequency error norm (HFEN, low value=high consistency) (28), structure similarity index (SSIM, high value=high similarity) (29), mutual information (MI, high value=high similarity) and the nondiagonal terms of the correlation matrix (cross-correlation, CC, high value=high correlation). In addition, eleven regions of interest (ROI) were evaluated locally, as described in (26), using ROI means, standard deviations, and RMSEs with respect to COSMOS as outcome measures.

In vivo 3T data: From a $3 \mathrm{~T}$ MRI system - Siemens Trio, 32-channel head-array, 1-mm ${ }^{3}$ isotropic resolution, fully-sampled acquisition (no parallel-imaging acceleration), 240×192×120 matrix, flip angle $=25^{\circ}, \mathrm{TE} / \mathrm{TR}=24.8 / 35 \mathrm{~ms}$, bandwidth $=100 \mathrm{~Hz} / \mathrm{pixel}, \mathrm{T}_{\text {acq }}=13: 30 \mathrm{~min}$. Phase unwrapping and background subtraction were performed with Laplacian (30) and Laplacian boundary value (31) (LBV) methods, respectively.

In vivo 7T data: From a 7T MRI system - Siemens MAGNETOM, 32-channel Nova head-array, $\mathrm{TE} / \mathrm{TR}=9 / 20 \mathrm{~ms}$, flip angle $=10^{\circ}$, bandwidth=120 Hz/pixel, fully-sampled $504 \times 608 \times 88$ matrix and $0.33 \times 0.33 \times 1.25-\mathrm{mm}^{3}$ voxel size $\left(\mathrm{T}_{\mathrm{acq}}=17: 30 \mathrm{~min}\right)$. Phase unwrapping and harmonic phase 
removal were performed using HARPERELLA (32) and VSHARP (33) $\left(\mathrm{R}_{0}=25 \mathrm{~mm}\right)$ algorithms, respectively.

Results were evaluated qualitatively in terms of artifact reduction and noise management.

Processing times were recorded for all experiments noting that, for the first phantom and second in vivo datasets, reconstructions were performed with Matlab 2016a (The Mathworks Inc., USA) on a laptop computer running an Intel $\mathrm{I} 76700 \mathrm{HQ}$ processor at $2.6 \mathrm{GHz}$ (3.5GHz Turbo) with 16GB RAM, whereas for all other experiments, Matlab 2014a was used on a desktop computer with an Intel i7-2600 processor at 3.40GHz and 32GB RAM. We used the MEDI toolbox for all NMEDI-related reconstructions (34), and we extended the fast TGV-QSM toolbox (35) to include the hereby-proposed formulation.

In phantom experiments, NMEDI's regularization parameter $(\lambda)$ was chosen to minimize RMSE. For in vivo reconstructions, free parameters were empirically selected. Default parameter values were used otherwise, including stopping criteria. For ADMM formulations, regularization parameters ( $\alpha_{1}$ and $\mu_{1}$, see Fig. S1) were optimized using the same criteria as in NMEDI. We also set a stopping change-rate threshold of $1 \%$ and a maximum of 50 iterations.

\section{Results}

Synthetic brain phantom: In low (or zero) magnitude regions, phase noise was approximately uniformly distributed. The linear solver handled such phase noise distributions poorly, yielding severe streaking artifacts (Fig. 1D). Nonlinear solvers, in contrast, resulted in two-orders-ofmagnitude RMSE improvements (Fig. 1E-G). Resulting global RMSE scores were: 834\% for TV, 25\% for TV-FANSI and 27\% for NMEDI. Regionally, focusing first on low-to-medium magnitude areas, e.g. the thalamic susceptibility gradient, nonlinear solvers showed improved noise management. Since simulated lesions were added to native susceptibility values, they overlapped with true structures (Fig. 1 and S3). TV-FANSI resolved such overlaps more accurately than NMEDI, i.e. it returned lower RMSE scores, though notably sphere-2 returned large error values for both reconstructions due to the larger relative influence of local structures. As expected, NMEDI with gradient-weighting returned a highly consistent piece-wise constant 
solution (Figure 1F), though as a consequence of edge-masking it also returned several inconsistencies at the tissue/lesion interface that were not present with conventional (unweighted) regularization (Figure $1 \mathrm{E}$ and $1 \mathrm{G}$ ). Overall, all nonlinear algorithms were robust to $2 \pi$ phase jumps (Fig. 1E-G), whereas the linear solution was corrupted by strong streaking (Fig. 1D). In terms of computational speed, TV-FANSI (12s) was approximately 8.5 times faster than NMEDI (104s).

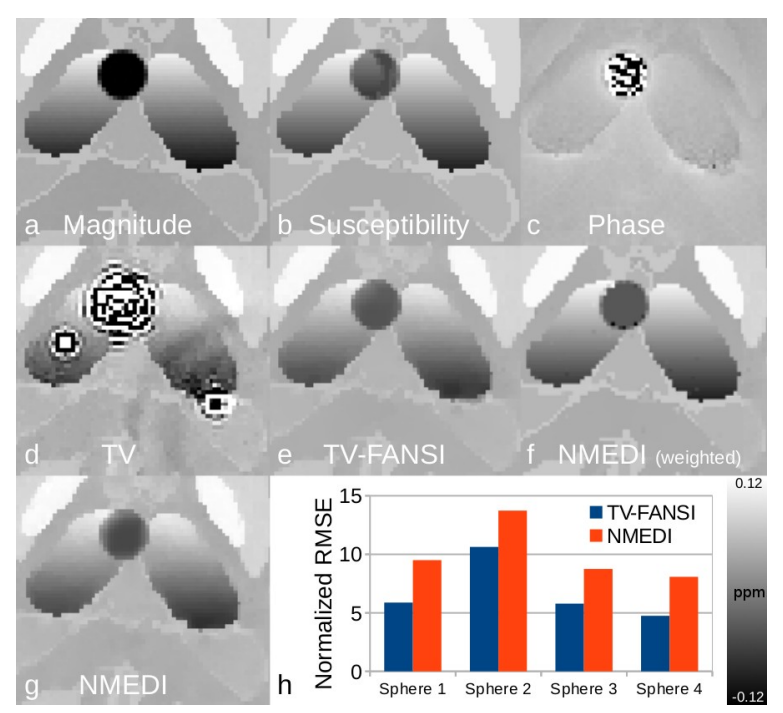

Fig. 1: Close-up detail of simulated data and reconstructions for sphere 2. Two $2 \pi$ phase jumps are also present in the simulated phase. a) Simulated magnitude, b) ground-truth susceptibility, c) simulated corrupted phase, d) TV, $\left.\alpha_{1}=2 e-4, \mu_{1}=100 \alpha_{1}, e\right)$ TV-FANSI, $\left.\alpha_{1}=2 e-4, \mu_{1}=100 \alpha_{1}, f\right)$ NMEDI (with gradient-weighting), and g) NMEDI (without gradient weighting) results, $\lambda=1750$. h) RMSE scores for each simulated (spherical) lesion.

COSMOS-brain simulation: The experiments (results summarized in Table 1) revealed that all algorithms performed similarly on all local measurements and global quality metrics. From visual inspection, we noted greater noise amplification on linear solutions and slight degradation across outer cortical regions (red arrows in Figure 2). RMSE between TV and TV-FANSI was 23\%, whereas between TV-FANSI and NMEDI the computed RMSE was 33\%. The sharpest features in such regions were obtained with NMEDI (putatively due to gradient-weighting), particularly venous structures (blue arrows), though staircase effects were also present. RMSE between TV-FANSI and NMEDI without gradient-weighting was 13\% (Fig. S4). In terms of 
computational time, ADMM-based formulations were notably faster than NMEDI; TV-FANSI, for example, was 35 times faster. Both linear and nonlinear ADMM solvers yielded similar (within 10\%) processing times per iteration.

Table 1. Local measurements (mean value, standard deviation and local RMSE, in ppb), quality metric performances and computation times (in seconds) for the COSMOS-based phantom.

\begin{tabular}{|l|c|c|c|c|}
\cline { 2 - 5 } \multicolumn{1}{l|}{ Caudate Nuc. } & TV & TV-FANSI & NMEDI & COSMOS \\
Putamen & $86 \pm 10[9]$ & $61 \pm 12[9]$ & $59 \pm 10[9]$ & $62 \pm 11$ \\
Globus Pallidus & $191 \pm 18[17]$ & $189 \pm 16[17]$ & $189 \pm 25[17]$ & $189 \pm 26$ \\
Red Nuc. & $87 \pm 13[11]$ & $86 \pm 12[12]$ & $86 \pm 14[12]$ & $90 \pm 16$ \\
Subs. Nigra & $163 \pm 21[17]$ & $162 \pm 20[17]$ & $161 \pm 23[21]$ & $174 \pm 24$ \\
Dent. Nuc. & $112 \pm 12[13]$ & $108 \pm 12[15]$ & $103 \pm 12[19]$ & $118 \pm 13$ \\
Genu (CC) & $-20 \pm 3[5]$ & $-19 \pm 3[5]$ & $-19 \pm 2[5]$ & $-22 \pm 4$ \\
Splenium (CC) & $-15 \pm 9[7]$ & $-15 \pm 9[6]$ & $-18 \pm 5[3]$ & $-17 \pm 5$ \\
Capsula Int. & $-51 \pm 9[13]$ & $-49 \pm 9[12]$ & $-45 \pm 8[10]$ & $-43 \pm 8$ \\
Frontal WM & $-17 \pm 6[7]$ & $-17 \pm 5[6]$ & $-13 \pm 3[4]$ & $-16 \pm 5$ \\
Occ WM & $-23 \pm 9[12]$ & $-27 \pm 7[8]$ & $-31 \pm 4[10]$ & $-30 \pm 10$ \\
\hline GM & $119 \pm 53[13]$ & $117 \pm 54[13]$ & $116 \pm 55[15]$ & $120 \pm 55$ \\
WM & $-29 \pm 17[10]$ & $-29 \pm 16[9]$ & $-28 \pm 14[8]$ & $-28 \pm 13$ \\
GM+WM & $87 \pm 77[12]$ & $86 \pm 77[12]$ & $85 \pm 77[13]$ & $88 \pm 78$ \\
\hline RMSE & 41.4 & 38.9 & 41.5 & - \\
HFEN & 37.5 & 35.1 & 33.9 & - \\
SSIM [x100] & 90.4 & 90.4 & 90.1 & - \\
MI [x100] & 53.0 & 55.2 & 54.5 & - \\
CC [x100] & 91.1 & 92.2 & 91.1 & - \\
\hline T [s] & 9.2 & 15 & 526 & - \\
\hline
\end{tabular}




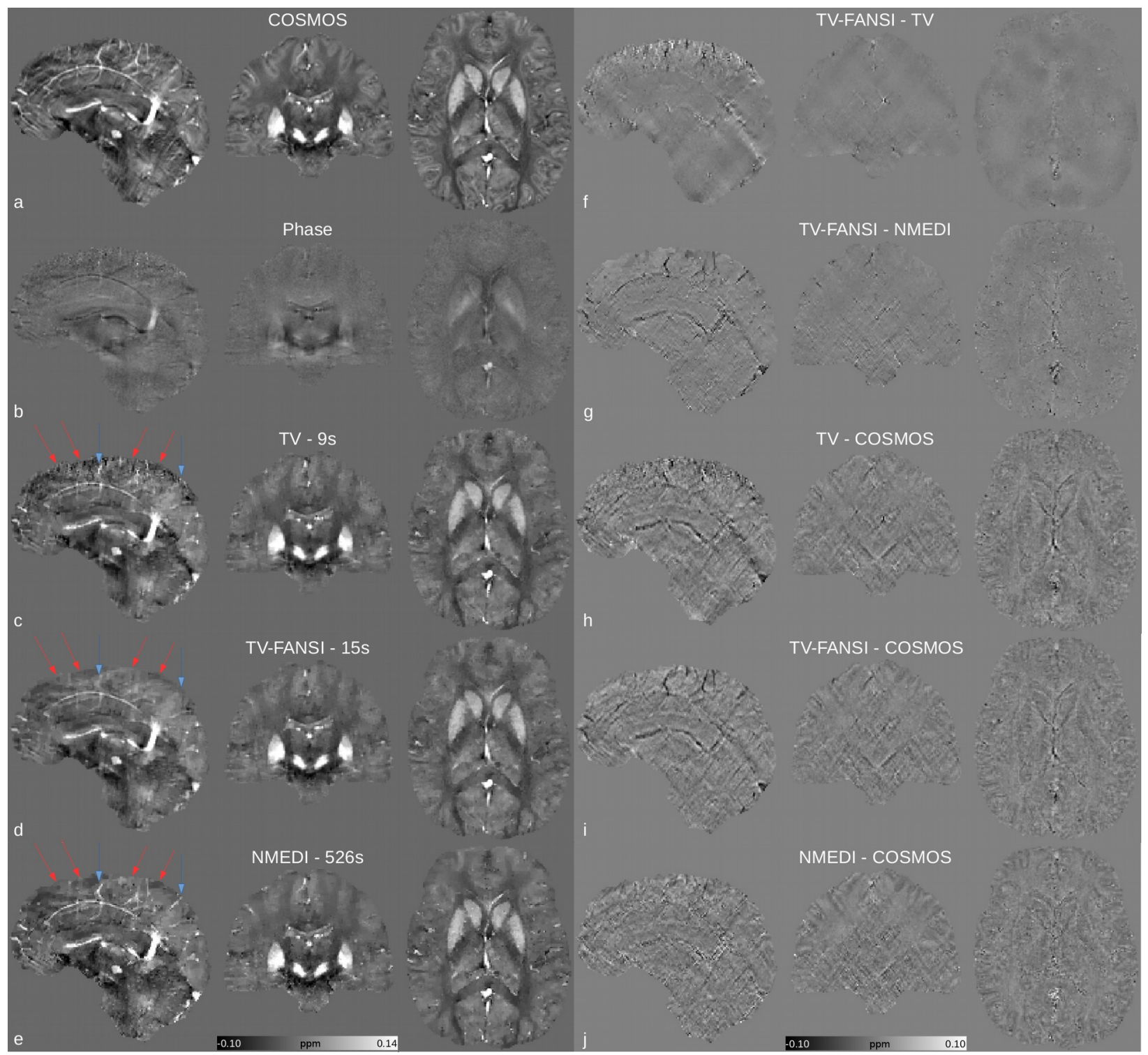

Fig. 2: Reconstruction results for the COSMOS phantom, in ppm. TV: $\alpha_{1}=4 e-3, \mu_{1}=16 \alpha_{1}, T V-$ FANSI: $\alpha_{1}=4 e-3, \mu_{1}=16 \alpha_{1}$ and NMEDI: $\lambda=60$.

Since the parameters for each algorithm were optimized for minimum RMSE, we also performed a stability test (Table 2 ) by modifying the regularization weight by $\pm 10 \%$. 
Table 2. Stability test results ( $\pm 10 \%$ variation in regularization parameter). Results provided are relative percentage changes, compared to the chosen optimal parameters.

\begin{tabular}{|l|cc|cc|}
\cline { 2 - 5 } \multicolumn{1}{c|}{} & \multicolumn{2}{c|}{ TV-FANSI } & \multicolumn{2}{c|}{ NMEDI } \\
\hline$\triangle \mathrm{RMSE}$ & 0.7 & 0.8 & 0.6 & 0.6 \\
$\Delta \mathrm{HFEN}$ & -2.9 & 3.7 & 4.1 & -2.9 \\
$\triangle \mathrm{SSIM}$ & -0.4 & $<0.1$ & $<0.1$ & -0.2 \\
$\Delta \mathrm{MI}$ & 0.5 & -0.9 & -5.1 & 0.5 \\
$\Delta \mathrm{CC}$ & -0.2 & -0.1 & -1.7 & $<0.1$ \\
\hline
\end{tabular}

In vivo data: Figure 3 shows representative cuts for difference maps across solutions obtained by empirically fine-tuning the free parameters. For 3T data, the optimal linear reconstruction was severely impaired by streaking artifacts (see arrows in Figure 3, and extended results in Fig. S5), which were greatly mitigated with nonlinear approaches. These observations were supported by the difference maps - streaking artifacts were most apparent in the TV-FANSI-TV differential (RMSE: 56.9\%), whereas TV-FANSI-NMEDI differences were relatively small (RMSE: 45.3\%). For $7 \mathrm{~T}$ data, greater differences were identified in cortical areas where e.g. the linear algorithm yielded much larger errors. TV-FANSI and NMEDI, in contrast, generated similar results in deep brain structures although notably, NMEDI was more effective in constraining artifacts emanating from boundary regions (Fig. S6).

In terms of processing time, ADMM-based methods were more than an order of magnitude faster than NMEDI. TV-FANSI, for example, was 31 times faster than NMEDI (54 s versus $1666 \mathrm{~s}$ ) for 3T data, and 79 times faster (224 s versus $17786 \mathrm{~s}$ ) for $7 \mathrm{~T}$ data. 


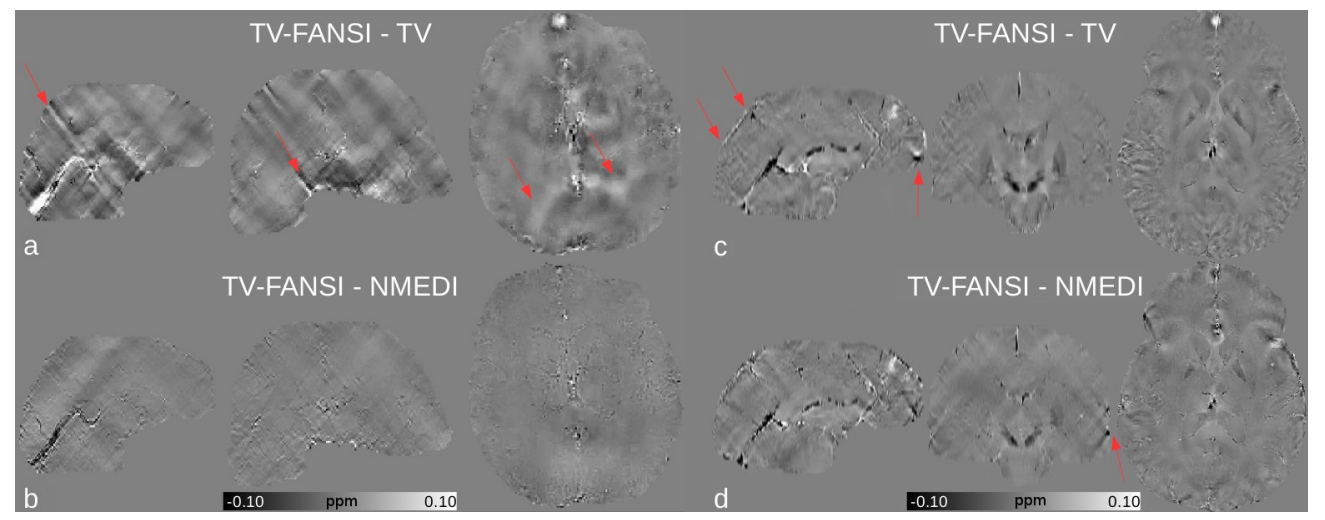

Fig. 3: Difference maps, in ppm, for the in-vivo data. $3 T$ data ( $a$ and b) parameters: TV: $\alpha_{1}=3.2 e-2, \mu_{1}=100 \alpha_{1}$, TV-FANSI: $\alpha_{1}=2 e-3, \mu_{1}=5 \alpha_{1}$ and NMEDI: $\lambda=260$. 7 T data (c and d) parameters: TV: $\alpha_{1}=6.3 e-3, \mu_{1}=25 \alpha_{1}$ TV-FANSI: $\alpha_{1}=1.6 e-4, \mu_{1}=25 \alpha_{1}$ and NMEDI: $\lambda=211$.

\section{Discussion}

Liu et al. (19) previously proposed a nonlinear model for QSM inversion known as NMEDI (nonlinear morphology-enabled dipole inversion) that included a data fidelity term in the image domain and a spatially-constrained total variation regularizer that overall promoted piece-wise constant solutions while sharing edges with the magnitude image. Liu et al.'s implementation included a relatively fast quasi-Newton fixed-point method, though to calculate solution updates the algorithm relies on linear matrix inversions with a slow-converging conjugate gradient solver. While effective, this approach is time-consuming. We hereby propose an ADMM algorithm that provides a framework to substantially accelerate QSM reconstructions without loss of accuracy with respect to the high-performance NMEDI approach. This was enabled through conversion of the nonlinear data-fidelity constraint into a simple, voxel-wise decoupled problem that can be solved rapidly. Regarding the additional variable introduced by the ADMM formalism, it should be highlighted that the penalty parameter in the nonlinear subproblem, $\mu$, was set to unity in all the experiments. Regularization parameters are discussed in the supplementary material.

Compared to NMEDI, the proposed nonlinear algorithm with TV regularization was 8.5 to 79 times faster depending on data type. Processing times were similar to those using a linear formulation (less than 20\% time increase per iteration).

In terms of image quality, the proposed nonlinear solver represents an improvement over linear formulations in terms of noise modeling and in preventing streaking artifacts emanating from 
zero or low SNR regions. Relative to NMEDI, TV-FANSI yielded similar reconstructions with a small systematic advantage for TV-FANSI in our analytic experiments and possibly slightly better artifact management for NMEDI in the high-resolution in vivo experiment (Fig. 3). Overall, however, differences between nonlinear methods were small, only slightly more marked in small-scale features with strong susceptibility gradients and low SNR, e.g. the venous vasculature. This can be explained by the spatially-varying weighting scheme that NMEDI applies to preserve anatomical edges (aided by edge information in magnitude data). Incorporating such prior knowledge from magnitude data would also help the proposed formulation better preserve such features, which warrants a future systematic investigation, as it would be studying in greater detail sources of error resulting from e.g. coil-combination, multiecho fitting or unwrapping errors. The high-resolution 7T experiment in this study explicitly highlighted that the present formulation must be extended to attenuate streaking artifacts emanating from boundary voxels - artifacts possibly generated by high-contrast dipole truncations, background filtering or other localized errors.

In addition to the benefits of faster convergence, the ADMM framework provides a solution to the nonlinear functional that is largely independent of the choice of regularizer. The same algorithm structure could thus be used for other variational penalties (as shown in the supplementary material with the TGV regularizer).

Finally, it is worth highlighting that the proposed algorithm, hereby initialized with background filtered field maps, could be extended to enable highly desirable single-step reconstructions directly from raw gradient-echo phase data $(16,36)$, which also warrants a future investigation.

\section{Conclusions}

The proposed FANSI algorithm provides a substantial gain in computational efficiency compared to NMEDI (up to 79 times faster) with similar performance in selected ROIs. Importantly, since morphological constraints are clearly beneficial for streaking control, the assessment of spatial weights for TV-FANSI warrants a subsequent investigation. Future directions also include singlestep formulations through incorporation of phase unwrapping and background removal into the functional and suitable preconditioning. 


\section{Acknowledgments}

We thank FONDECYT 1161448, CONICYT Programa PIA-Anillo ACT1416, and Becas de Doctorado Nacional CONICYT, Folio 21150369, for their funding support. JAC is supported by core funding from the Wellcome, UK [203147/Z/16/Z]. We also thank Hendrik Mattern and Oliver Speck (BMMR, Magdeburg) for providing the 7T dataset. 


\section{References}

1. Salomir R, de Senneville BD, Moonen CT. A fast calculation method for magnetic field inhomogeneity due to an arbitrary distribution of bulk susceptibility. Concepts Magn Reson Part B 2003;19B:26-34.

2. Marques JP, Bowtell R. Application of a Fourier-based method for rapid calculation of field inhomogeneity due to spatial variation of magnetic susceptibility. Concepts Magn Reson Part B 2005;25B:65-78.

3. Wharton S, Schäfer A, Bowtell R. Susceptibility mapping in the human brain using thresholdbased k-space division. Magn Reson Med 2010;63:1292-1304.

4. Shmueli K, de Zwart J a, van Gelderen P, Li T-Q, Dodd SJ, Duyn JH. Magnetic susceptibility mapping of brain tissue in vivo using MRI phase data. Magn Reson Med 2009;62:1510-1522.

5. de Rochefort L, Brown R, Prince MR, Wang Y. Quantitative MR susceptibility mapping using piece-wise constant regularized inversion of the magnetic field. Magn Reson Med 2008;60:1003-1009.

6. Kressler B, Rochefort L De. Nonlinear regularization for per voxel estimation of magnetic susceptibility distributions from MRI field maps. IEEE Trans Med Imaging 2010;29:273-281. 7. Liu T, Liu J, De Rochefort L, Spincemaille P, Khalidov I, Ledoux JR, Wang Y. Morphology enabled dipole inversion (MEDI) from a single-angle acquisition: Comparison with COSMOS in human brain imaging. Magn Reson Med 2011;66:777-783.

8. Liu J, Liu T, De Rochefort L, Ledoux J, Khalidov I, Chen W, Tsiouris AJ, Wisnieff C, Spincemaille P, Prince MR, et al. Morphology enabled dipole inversion for quantitative susceptibility mapping using structural consistency between the magnitude image and the susceptibility map. Neuroimage 2012;59:2560-2568.

9. Bilgic B, Fan AP, Polimeni JR, Cauley SF, Bianciardi M, Adalsteinsson E, Wald LL, Setsompop K. Fast quantitative susceptibility mapping with L1-regularization and automatic parameter selection. Magn Reson Med 2014;72:1444-1459.

10. de Rochefort L, Liu T, Kressler B, Liu J, Spincemaille P, Lebon V, Wu J, Wang Y. Quantitative susceptibility map reconstruction from MR phase data using bayesian regularization: validation and application to brain imaging. Magn Reson Med 2010;63:194-206. 11. Wu B, Li W, Guidon A, Liu C. Whole brain susceptibility mapping using compressed sensing. Magn Reson Med 2012;67:137-147. 
12. Wang S, Liu T, Chen W, Spincemaille P, Wisnieff C, Tsiouris a J, Zhu W, Pan C, Zhao L, Wang Y. Noise Effects in Various Quantitative Susceptibility Mapping Methods. IEEE Trans Biomed Eng 2013;60:3441-3448.

13. Bredies K, Kunisch K, Pock T. Total Generalized Variation. SIAM J Imaging Sci 2010;3:492-526

14. Knoll F, Bredies K, Pock T, Stollberger R. Second order total generalized variation (TGV) for MRI. Magn Reson Med 2011;65:480-491.

15. Yanez F, Fan A, Bilgic B, Milovic C, Adalsteinsson E, Irarrazaval P. Quantitative Susceptibility Map Reconstruction via a Total Generalized Variation Regularization. 2013 Int Workshop Pattern Recognit Neuroimaging 2013:203-206

16. Langkammer C, Bredies K, Poser B, Barth M, Reishofer G, Fan AP, Bilgic B, Fazekas F, Mainero C, Ropele S. Fast quantitative susceptibility mapping using 3D EPI and total generalized variation. Neuroimage 2015;111:622-630.

17. Bilgic B, Chatnuntawech I, Langkammer C, Setsompop K. Sparse Methods for Quantitative Susceptibility Mapping. Wavelets and Sparsity XVI, SPIE 2015. doi: 10.1117/12.2188535 18. Gudbjartsson H, Patz S. The Rician distribution of noisy MRI data. Magn Reson Med 1995;34:910-914.

19. Liu T, Wisnieff C, Lou M, Chen W, Spincemaille P, Wang Y. Nonlinear formulation of the magnetic field to source relationship for robust quantitative susceptibility mapping. Magn Reson Med 2013;69:467-476.

20. Zhao B, Setsompop K, Ye H, Cauley S. and Wald LL. Maximum likelihood reconstruction for magnetic resonance fingerprinting, IEEE Trans Med Imaging 2016;35:1812-1823. . 21. Goldstein T, Osher S. The split Bregman method for L1-regularized problems. SIAM J Imaging Sci 2009;2:323-343

22. Boyd S, Parikh N, Chu E, Peleato B, Eckstein J. Distributed Optimization and Statistical Learning via the Alternating Direction Method of Multipliers. Found Trends Mach Learn 2010;3:1-122.

23. Ramani S, and Fessler JA. Parallel MR Image Reconstruction Using Augmented Lagrangian Methods. IEEE Trans Med Imaging 2011;30:694-706.

24. Press WH, Teukolsky SA, Vetterling Wt, Flannery BP. Numerical Recipes in C: the art of scientific computing, Second Edition. Cambridge Univ. Press, New York. 1992. 
25. Wisnieff C, Liu T, Spincemaille P, Wang S, Zhou D, Wang Y. Magnetic susceptibility anisotropy: cylindrical symmetry from macroscopically ordered anisotropic molecules and accuracy of MRI measurements using few orientations. Neuroimage 2013;70:363-376. 26. Langkammer C, Schweser F, Shmueli K, Kames C, Li X, Guo L, Milovic C, Kim J, Wei H, Bredies K, Buch S, Guo Y, Liu Z, Meineke J, Rauscher A, Marques JP, Bilgic B. Quantitative Susceptibility Mapping: Report from the 2016 Reconstruction Challenge. Magn Reson Med 2017. doi: $10.1002 / \mathrm{mrm} .26830$

27. Liu T, Spincemaille P, De Rochefort L, Kressler B, Wang Y. Calculation of susceptibility through multiple orientation sampling (COSMOS): A method for conditioning the inverse problem from measured magnetic field map to susceptibility source image in MRI. Magn Reson Med 2009;61:196-204.

28. Saiprasad R, Bresler Y. MR image reconstruction from highly undersampled k-space data by dictionary learning. IEEE Trans Med Imaging 2011;30:1028-1041.

29. Wang Z, Bovik A, Sheikh H, Simoncelli E. Image quality assessment: from error visibility to structural similarity. IEEE Trans Image Process 2004;13:600-612.

30. Li W, Wu B, Liu C. Quantitative susceptibility mapping of human brain reflects spatial variation in tissue composition. Neuroimage 2011;55:1645-1656.

31. Zhou D, Liu T, Spincemaille P, Wang Y. Background field removal by solving the Laplacian boundary value problem. NMR Biomed 2014;27:312-319.

32. Li W, Avram A V, Wu B, Xiao X, Liu C. Integrated Laplacian-based phase unwrapping and background phase removal for quantitative susceptibility mapping. NMR Biomed. 2014;27:219227.

33. Fang J, Bao L, Li X, van Zijl P, Chen Z. Background field removal using a region adaptive kernel for quantitative susceptibility mapping of human brain. Journal of Magnetic Resonance. 2017;281:130-140. doi: doi.org/10.1016/j.jmr.2017.05.004..

34. Cornel MRI Research Lab. Quantitative Susceptibility Mapping. MEDI toolbox. http://weill.cornell.edu/mri/pages/qsm.html. Updated May 20, 2016.

35. Bilgic, B. Software. Fast Total Generalized Variation regularized QSM toolbox. http://martinos.org/ berkin/software.html. Updated November 2016.

36. Chatnuntawech I, McDaniel P, Cauley S.F, Gagoski BA, Langkammer C, Martin A, Grant P.E, Wald LL, Setsompop K, Adalsteinsson E, Bilgic B. TGV-Regularized Single-Step Quantitative Susceptibility Mapping. NMR Biomed 2017;30:e3570. 


\section{Fast Nonlinear Susceptibility Inversion with Variational Regularization}

Carlos Milovic, Berkin Bilgic, Bo Zhao, Julio Acosta-Cabronero, and Cristian Tejos

\section{Supplementary Material}

\section{ADMM solver for TV regularization (17)}

Let's take the $\chi$ subproblem of the augmented functional (Eq. 2), defined using the TV regularization term:

$$
\operatorname{argmin}_{\chi} \frac{\mu}{2} \cdot\left\|F^{H} D F \chi-z+s\right\|_{2}^{2}+\alpha_{1}|\nabla \chi|_{1}
$$

Eq. S1.1

Here we introduce additional variables following the ADMM formalism:

$$
\operatorname{argmin}_{\chi, z_{1}} \frac{\mu}{2} \cdot\left\|F^{H} D F \chi-z+s\right\|_{2}^{2}+\alpha_{1}\left|z_{1}\right|_{1}+\frac{\mu_{1}}{2}\left|\nabla \chi-z_{1}+s_{1}\right|_{2}^{2}
$$

Eq. S1.2

Firstly, we can solve the subproblem for $\chi$ :

$$
\operatorname{argmin}_{\chi} \frac{\mu}{2} \cdot\left\|F^{H} D F \chi-z+s\right\|_{2}^{2}+\frac{\mu_{1}}{2}\left|\nabla \chi-z_{1}+s_{1}\right|_{2}^{2}
$$

If we rewrite the gradient operators as linear operator in the Fourier domain by $\partial_{\mathrm{x}}=\mathrm{F}^{\mathrm{H}} \mathrm{E}_{\mathrm{x}} \mathrm{F}, \partial_{\mathrm{y}}=$ $\mathrm{F}^{\mathrm{H}} \mathrm{E}_{\mathrm{y}} \mathrm{F}, \partial_{\mathrm{z}}=\mathrm{F}^{\mathrm{H}} \mathrm{E}_{\mathrm{z}} \mathrm{F}$ and take the derivative w.r.t $\chi$ :

$$
\begin{gathered}
\mu F^{H} D^{H} F\left(F^{h} D F X-z+s\right)+\mu_{1} F^{h} E^{H} F\left(F^{H} E F X-z_{1}+s_{1}\right)=0 \\
\mu F^{H} D^{H} D F X-\mu F^{H} D^{H} F(z-s)+\mu_{1} F^{H} E^{H} E F X=\mu_{1} F^{H} E^{H} F\left(z_{1}-s_{1}\right)
\end{gathered}
$$

Here we apply the Fourier Transform to decouple terms, and then achieve:

$$
\chi=F^{H}\left[\frac{\mu D^{H} F(z-s)+\mu_{1} E^{H} F\left(z_{1}-s_{1}\right)}{\mu D^{H} D+\mu_{1} E^{H} E}\right]
$$

With $\mathrm{F} \chi$ known, we now solve the outer functional, for $\mathrm{z}_{1}$ and any other variable introduced by 
the ADMM formalism (for solving the data fidelity term).

The solution for $\mathrm{z}_{1}$ comes from the soft thresholding operation:

$$
z_{1}=\max \left(\left|\nabla \chi+s_{1}\right|-\alpha_{1} / \mu_{1}, 0\right) \cdot \operatorname{sign}\left(\nabla \chi+s_{1}\right) \quad \text { Eq. S1.7 }
$$

And the update rule for $\mathrm{s}_{1}$ is given by:

$$
s_{1}=s_{1}+\nabla \chi-z_{1} \quad \text { Eq. } S 1.8
$$

We iterate solving for $\mathrm{F} \chi$ and all the ADMM introduced variables until convergence is achieved, measured by the norm of the changes in $\chi$ between iterations.

The choice of $\mu_{1}$ used by the regularization subproblem is closely related to the regularization parameter, $\alpha_{1}$. A preliminary analysis revealed that optimal $\mu_{1} / \alpha_{1}$ ratios might be restricted to the $[1,100]$ range, which effectively narrows the search for optimal regularization parameters.

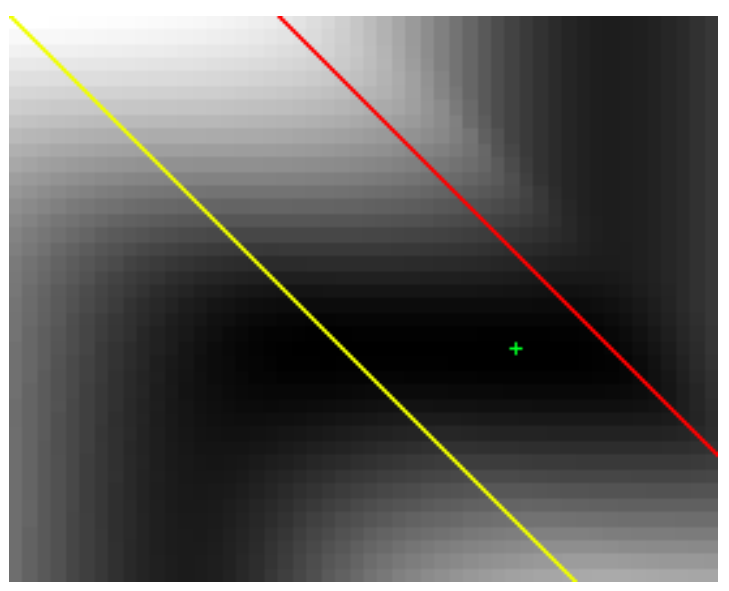

Figure S1. RMSE map for the $\mu_{1}$ and $\alpha_{1}$ parameters, using the COSMOS-brain data. The vertical axis represents $\alpha_{1}$ values (from $10^{-2}$ to $10^{-6}$, top-bottom) and the horizontal axis represents $\mu_{1}$ values (from 1 to $10^{-5}$, left-right). Step size is $10^{-0.1}$. The red line indicates $\mu_{1} / \alpha_{1}=1$. The yellow line indicates $\mu 1 / \alpha 1=100$. The green cross indicates the location of the global minimum error.

Similar results were found to those in Figure S1 with different levels of noise and other quality metrics.

Further investigations are warranted. 


\section{ADMM solver for TGV regularization (17)}

Let's take the $\chi$ subproblem of the augmented functional (Eq. 2), defined using the TGV regularization term:

Eq. S2.1

$$
\operatorname{argmin}_{\chi, v} \frac{\mu}{2} \cdot\left\|F^{H} D F \chi-z+s\right\|_{2}^{2}+\alpha_{1}|\nabla \chi-v|_{1}+\alpha_{0}|\epsilon(v)|_{1}
$$

Eq.

$$
\operatorname{argmin}_{\chi, v, z_{1}, z_{0}} \frac{\mu}{2} \| F^{H} D F \chi-z+\left.s\right|_{2} ^{2}+\alpha_{1}\left|z_{1}\right|_{1}+\frac{\mu_{1}}{2}\left|\nabla \chi-v-z_{1}+s_{1}\right|_{2}^{2}+\alpha_{0}\left|z_{0}\right|_{1}+\frac{\mu_{0}}{2}\left|\epsilon(v)-z_{0}+s_{0}\right|_{2}^{2}
$$

\section{S2.2}

Firstly, we can solve the subproblem for $\chi$ and v:

$$
\operatorname{argmin}_{\chi, v} \frac{\mu}{2} \cdot\left\|F^{H} D F \chi-z+s\right\|_{2}^{2}+\frac{\mu_{1}}{2}\left|\nabla \chi-v-z_{1}+s_{1}\right|_{2}^{2}+\frac{\mu_{0}}{2}\left|\epsilon(v)-z_{0}+s_{0}\right|_{2}^{2} \quad \text { Eq. S2.3 }
$$

If we rewrite the gradient operators as linear operator in the Fourier domain by $\partial_{x}=F^{H} E_{x} F, \partial_{y}=$ $\mathrm{F}^{\mathrm{H}} \mathrm{E}_{\mathrm{y}} \mathrm{F}, \partial_{\mathrm{z}}=\mathrm{F}^{\mathrm{H}} \mathrm{E}_{\mathrm{z}} \mathrm{F}$ and $\varepsilon=\mathrm{F}^{\mathrm{H}} \mathrm{SF}$, and take the derivative w.r.t $\chi$ :

$$
\begin{gathered}
\mu F^{H} D^{H} F\left(F^{H} D F X-z+s\right)+\mu_{1} F^{H} E^{H} F\left(F^{H} E F X-v-z_{1}+s_{1}\right)=0 \\
\mu F^{H} D^{H} D F X-\mu F^{H} D^{H} F(z-s)+\mu_{1} F^{H} E^{H} E F X-\mu_{1} F^{H} E^{h} F v=\mu_{1} F^{H} E^{H} F\left(z_{1}-s_{1}\right)
\end{gathered}
$$

Eq. S2.5

Here we apply the Fourier Transform to decouple terms:

$$
\left(\mu D^{H} D+\mu_{1} E^{H} E\right) F X-\mu_{1} E^{H} F v=\mu D^{H} F(z-s)+\mu_{1} E^{H} F\left(z_{1}-s_{1}\right)
$$

With the differentiation w.r.t. v, and following the same steps, we reach this system of equations: 


$$
\left[\begin{array}{cc}
\mu D^{H} D+\mu_{1} E^{H} E & -\mu_{1} E^{H} \\
-\mu_{1} E^{H} & \mu_{1}+\mu_{0} S^{H} S
\end{array}\right]\left[\begin{array}{c}
F X \\
F v
\end{array}\right]=\left[\begin{array}{l}
\mu D^{H} F(z-s)+\mu_{1} E^{H} F\left(z_{1}-s_{1}\right) \\
\mu_{0} S^{H} F\left(z_{0}-s_{0}\right)-\mu_{1} F\left(z_{1}-s_{1}\right)
\end{array}\right]
$$

which may be solved pixel by pixel by inversion of the left-hand side matrix. We use Cramer's rule for this matrix inversion. Since all the elements in the matrix do not depend on the ADMM variables, it may be precomputed, and used without modification in all iterations.

With $\mathrm{F}_{X}$ and $\mathrm{Fv}$ known, we now solve the outer functional, for $\mathrm{z}_{1}, \mathrm{z}_{0}$ and any other variable introduced by the ADMM formalism (for solving the data fidelity term).

The solution for $\mathrm{z}_{1}$ and $\mathrm{z}_{0}$ come from the soft thresholding operation:

$$
\begin{array}{r}
z_{1}=\max \left(\left|\nabla \chi-v+s_{1}\right|-\alpha_{1} / \mu_{1}, 0\right) \cdot \operatorname{sign}\left(\nabla \chi-v+s_{1}\right) \\
z_{0}=\max \left(\left|\epsilon(v)+s_{0}\right|-\alpha_{0} / \mu_{0}, 0\right) \cdot \operatorname{sign}\left(\epsilon(v)+s_{0}\right)
\end{array}
$$

And the update rule for $\mathrm{s}_{1}$ and $\mathrm{s}_{0}$ is given by:

$$
\begin{array}{ll}
s_{1}=s_{1}+\nabla X-v-z_{1} & \text { Eq. } S 2.10 \\
s_{0}=s_{0}+\epsilon(v)-z_{0} & \text { Eq. } S 2.11
\end{array}
$$

We iterate solving for $\mathrm{F} \chi, \mathrm{Fv}$ and all the ADMM introduced variables until convergence is achieved, measured by the norm of the changes in $\chi$ between iterations. 


\section{Extended TV-FANSI results}

Synthetic brain phantom. All the simulated lesions are displayed in axial cuts of the synthetic phantom (Figure S2). Susceptibility values, from left to right, -0.5, -0.3, 0.6 and $1.2 \mathrm{ppm}$.

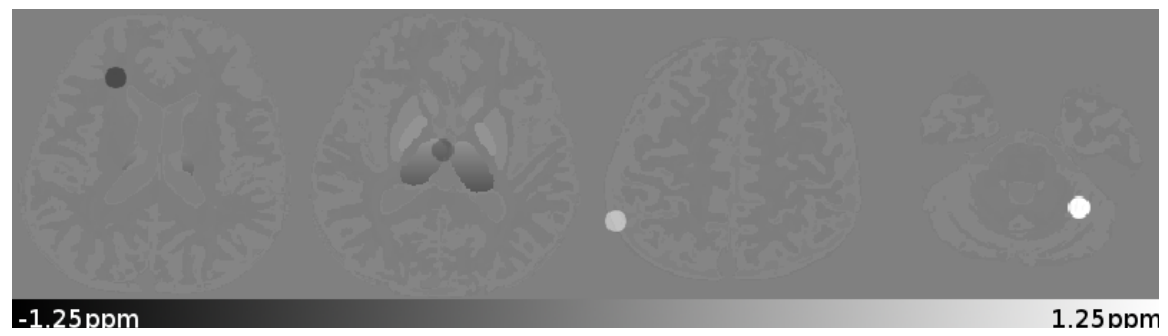

Figure S2. Axial cuts that shows all four simulated lesions. 
Figure S3 shows the results achieved for the synthetic phantom.

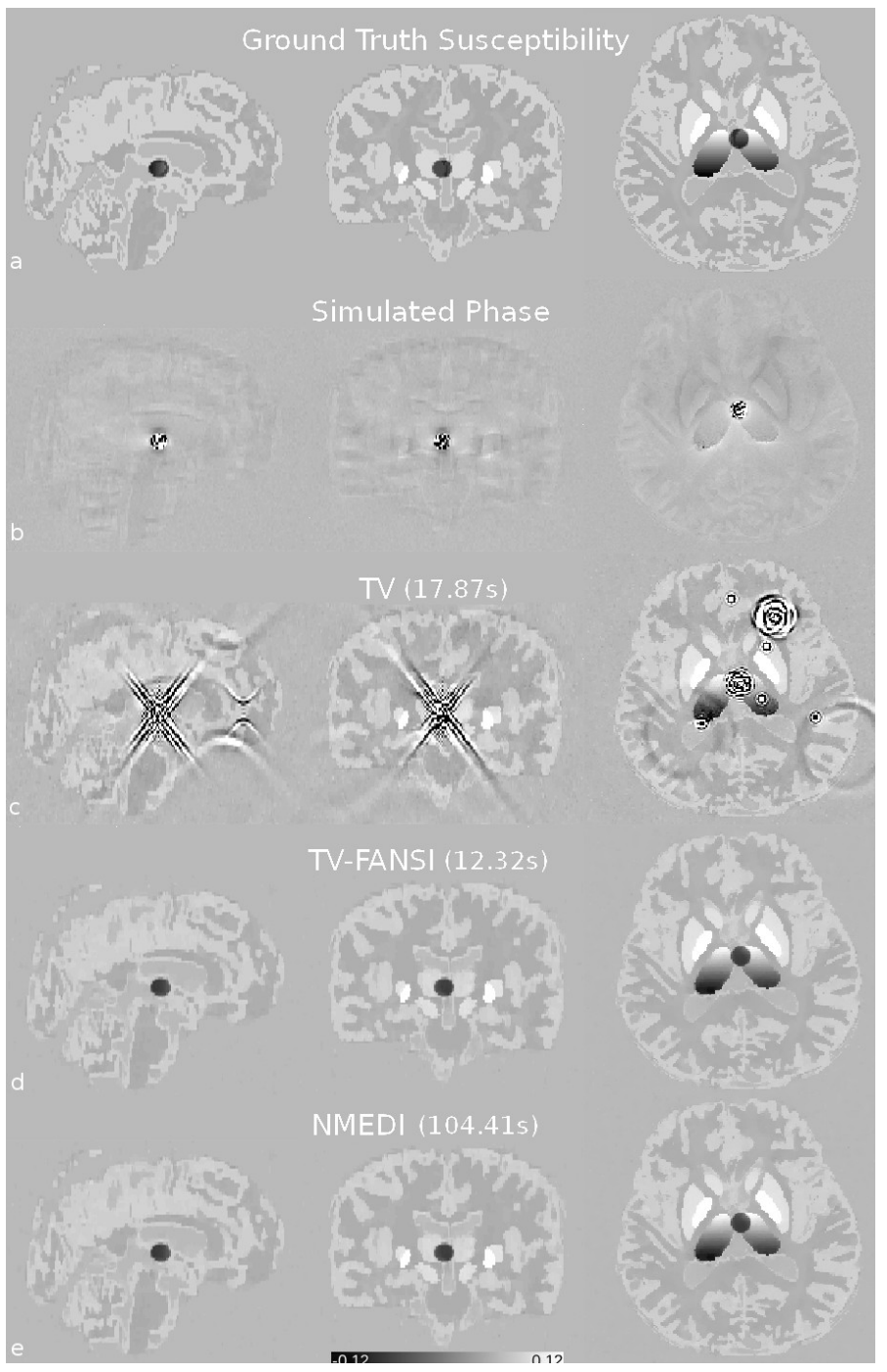

Figure S3. Synthetic brain phantom with simulated lesion and results. a) Ground truth susceptibility. b) Simulated phase. c) TV result. d) TV-FANSI result. e) NMEDI result (without gradient weighting). 
COSMOS-brain. Additional results and difference maps are presented in Figure S4, to provide further information about NMEDI's gradient weighting impact.

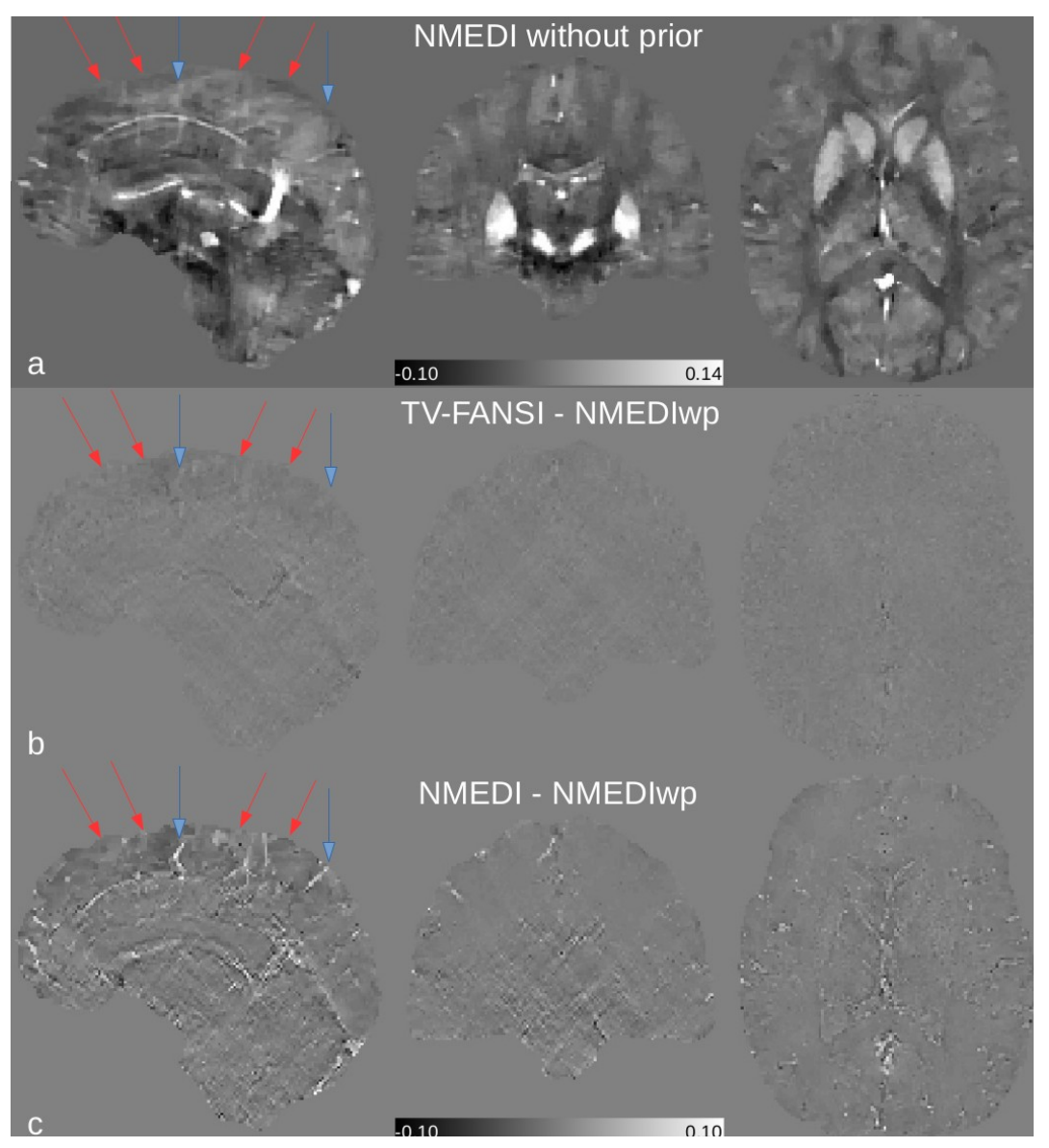

Figure S4. COSMOS-brain additional results results. a) NMEDI result without gradient weighting algorithm. b) Difference map between TV-FANSI and NMEDI without the gradient prior. c) Difference map using the NMEDI algorithm with and withouth gradient weighting. 
In vivo data. Reconstruction results are provided in Figure S5 and S6, along with the input local field (in ppm).

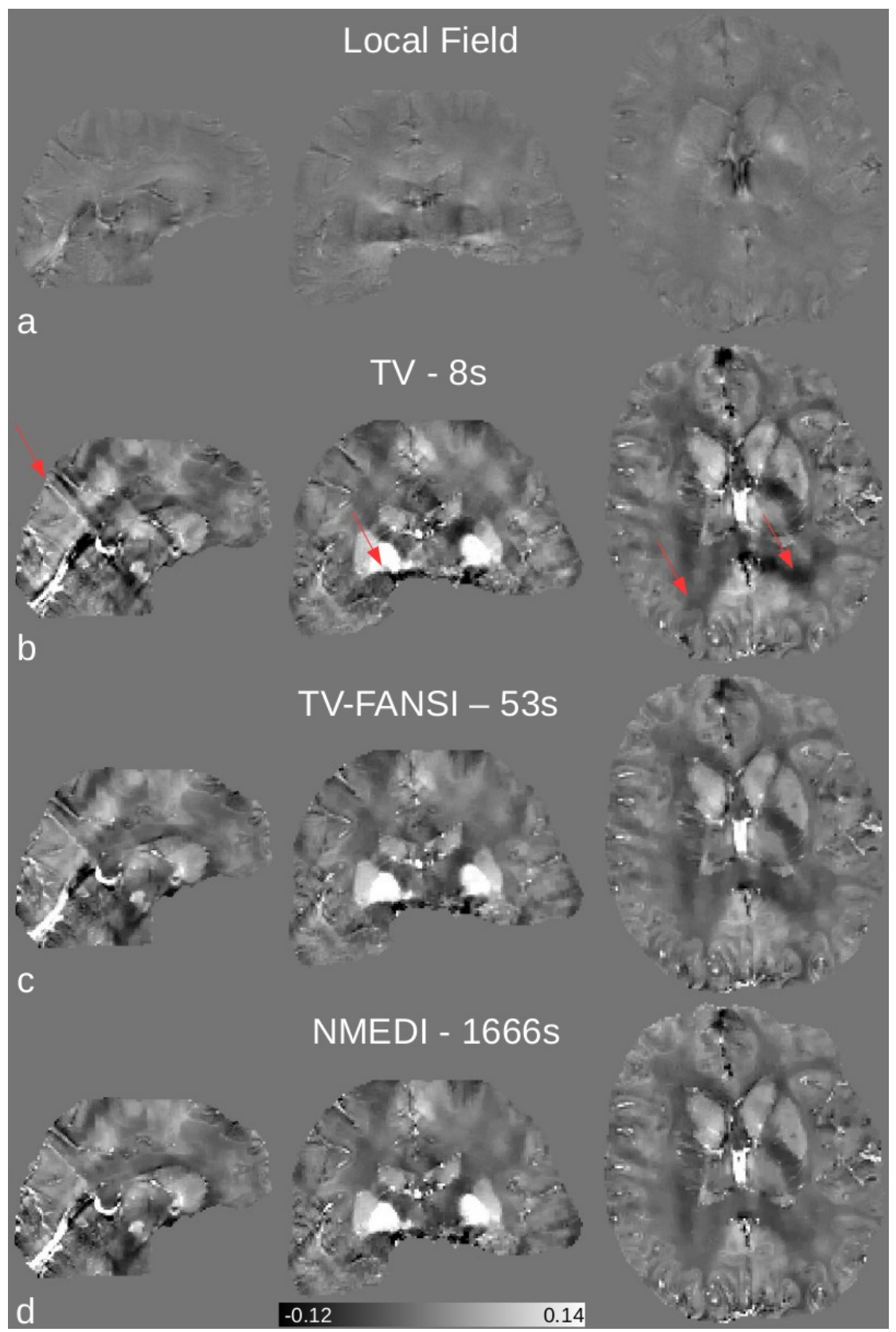

Fig. S5: Results for the 3T in-vivo data. a) Local field used as input. b) TV, c) TV-FANSI and d) NMEDI results. 


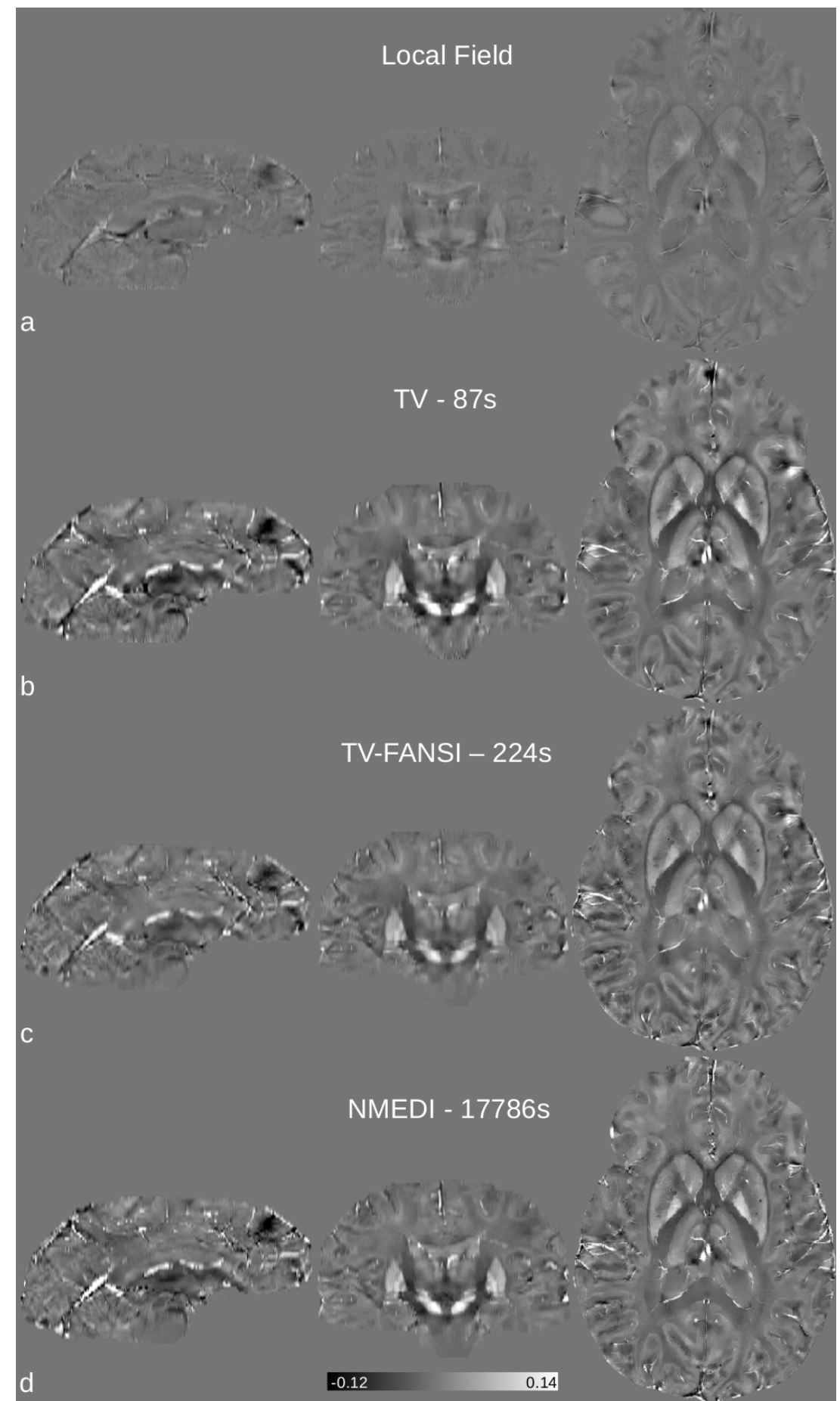

Fig. S6: Results for the 7T in-vivo data. a) Local field used as input. b) TV, c) TV-FANSI and d) NMEDI results. 


\section{Results using TGV regularization}

As in the convention used in the main text, we referred to ADMM-based linear formulation as "TGV", and used "TGV-FANSI" to refer to the nonlinear counterpart.

Synthetic brain phantom. Solutions using TGV regularization are shown in Figure S7 (same cuts than those shown in Figure S3). Magnified views (Figure 1 extension) are also shown in Figure S8. TGV yielded RMSE of $830.58 \%$, whereas TGV-FANSI scored $24.84 \%$. As with TVFANSI, TGV-FANSI improved NMEDI's performance in this experiment. Both, TV-FANSI and TGV-FANSI returned similar errors (RMSE scores for TGV-FANSI were within $0.3 \%$ relative to those for TV-FANSI, and signified an improvement of $1.7 \%$ with respect to NMEDI), with TVFANSI operating 2.5x per iteration faster than TGV-FANSI but TGV-FANSI, with 34.05s, was 3.1x faster than NMEDI. RMSE values for each simulated lesion are within $0.0025 \%$ of variation compared to TV-FANSI.

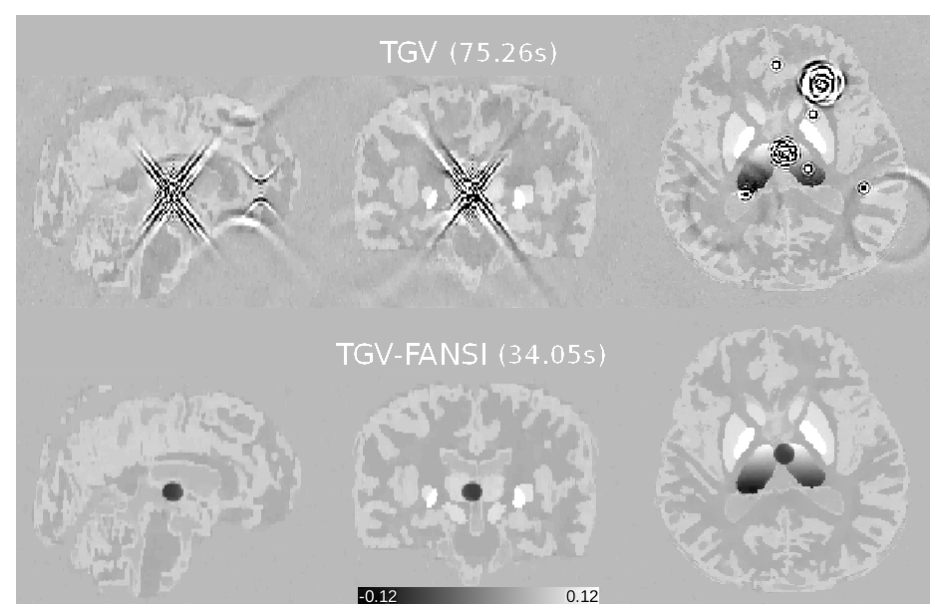

Fig. S7: TGV results for the synthetic brain phantom with simulated lesions. 


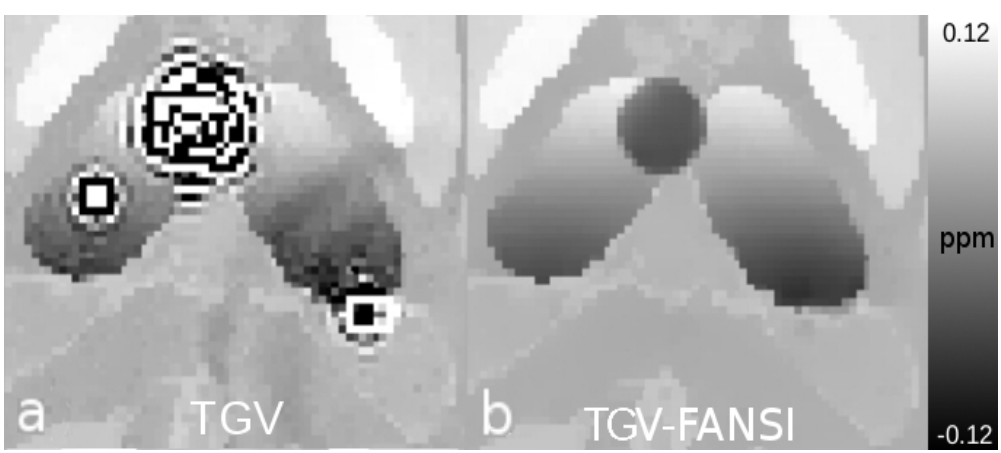

Fig. S8: Magnified view of the reconstructions around sphere 2 . Two $2 \pi$ phase jumps are also present in the simulated thalamus. a) TGV result. b) TGV-FANSI result. 
COSMOS-brain. Quality metrics for TGV-FANSI slightly improved those for TV-FANSI (RMSE: 39.0169, HFEN 34.3272, SSIM: 0.9038, MI: 0.5577, CC: 0.912). Results are displayed in Figures S9 and S10. TGV-FANSI required a greater number of iterations than linear TGV (42 vs. 17) but was 6.7 times faster than NMEDI. Local measurements were largely equivalent to those achieved by TV-FANSI, as shown in Table S1. Finally, an extended stability test revealed consistent results to those achieved by TV-FANSI.

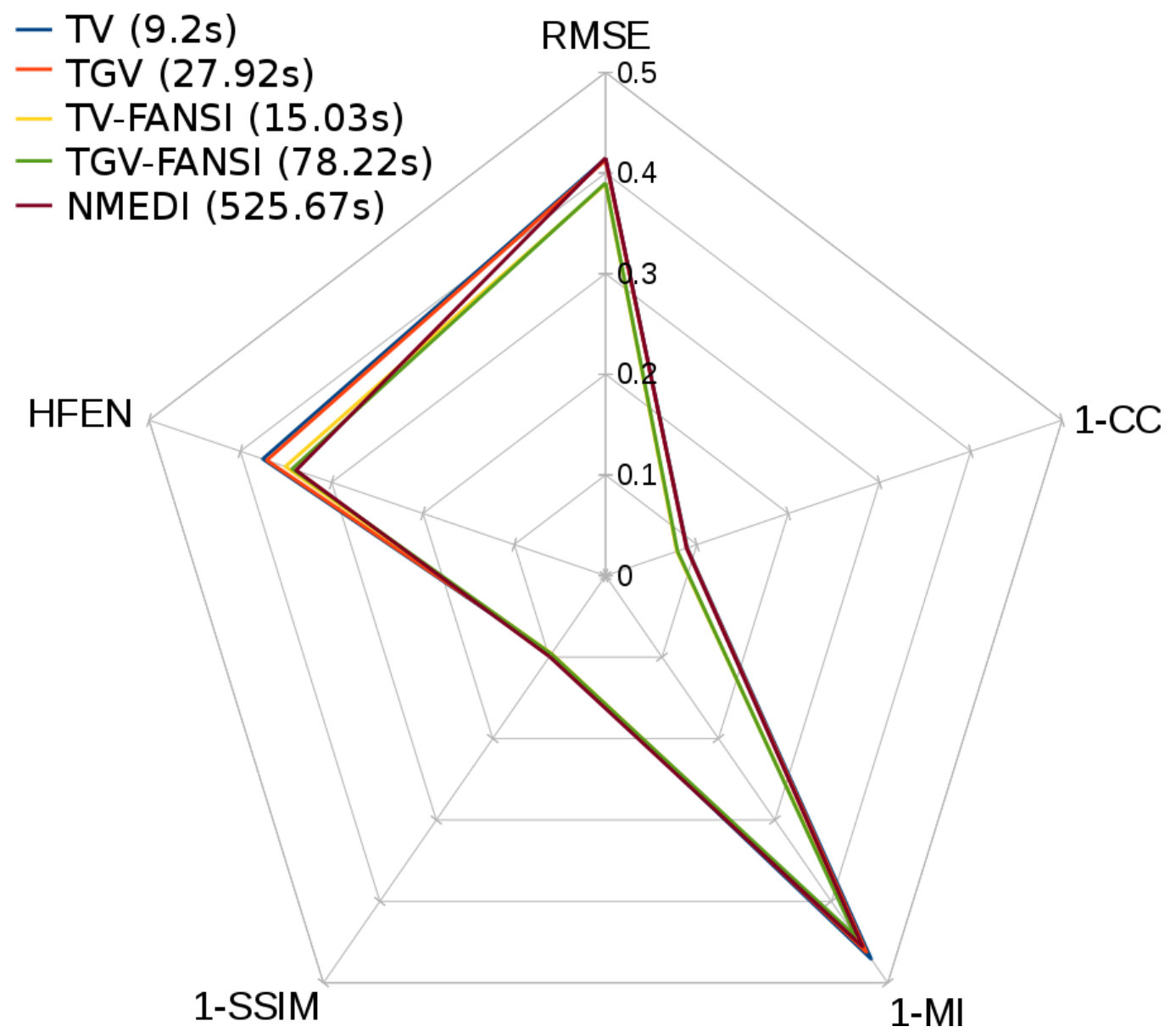

Fig. S9: Normalized quality scores for the COSMOS phantom results. In this graph, lower scores indicate better performance. 


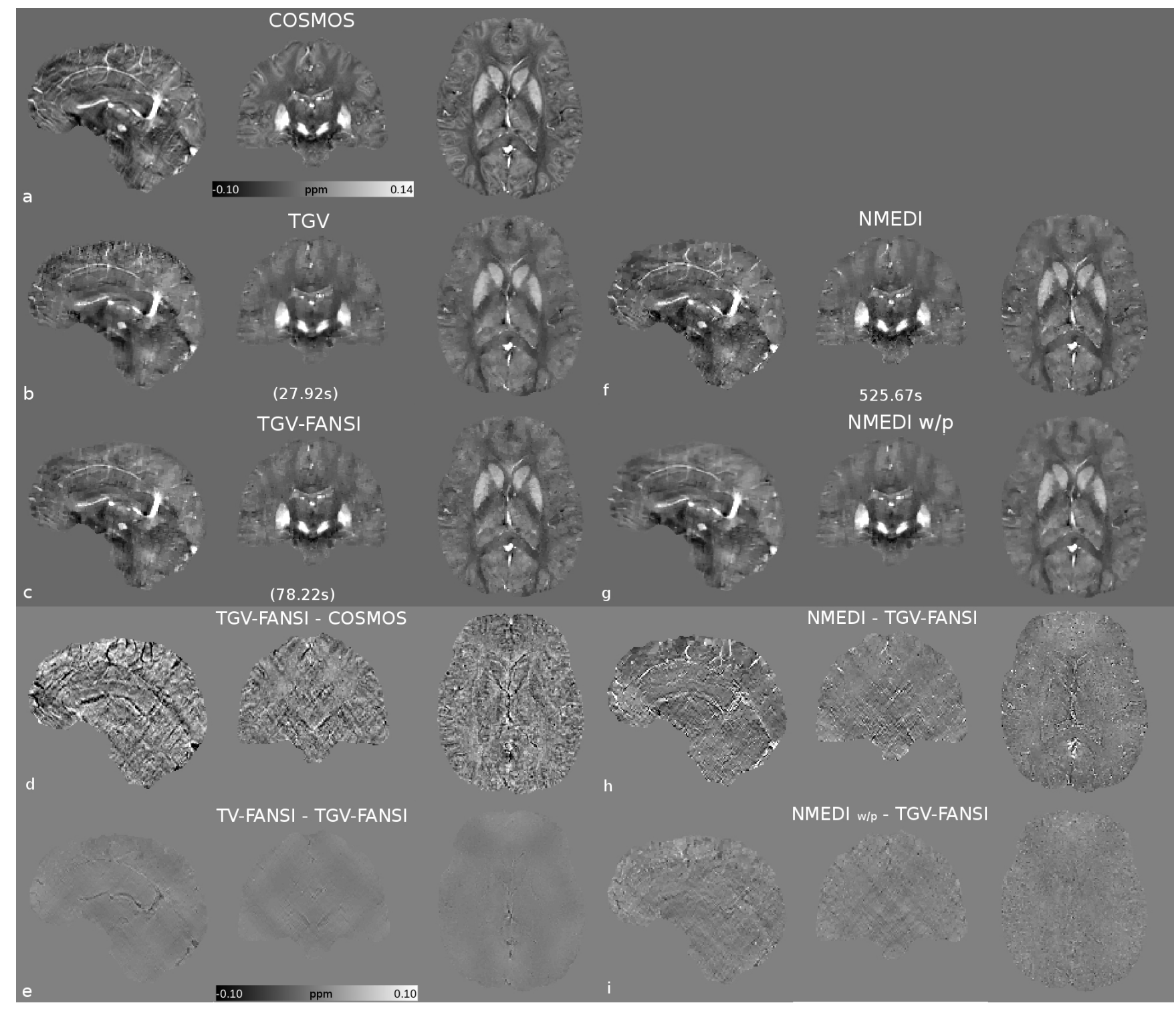

Fig. S10: Reconstruction results for the COSMOS phantom, using the TGV regularization. Additional difference maps with NMEDI without edge information prior are displayed. 
Table S1. Complete local ROI measurements (mean value, standard deviation and local RMSE compared to COSMOS [], in ppb).

\begin{tabular}{|l|c|c|c|c|c|c|}
\cline { 2 - 7 } \multicolumn{1}{c|}{ Caudate Nuc. } & NMEDI & TV & TV-FANSI & TGV & TGV-FANSI & COSMOS \\
\hline Putamen & $83 \pm 13[10]$ & $66 \pm 10[9]$ & $61 \pm 12[9]$ & $66 \pm 9[9]$ & $61 \pm 12[9]$ & $62 \pm 11$ \\
Globus Pallidus & $189 \pm 25[17]$ & $191 \pm 18[9]$ & $85 \pm 13[9]$ & $85 \pm 13[9]$ & $84 \pm 14[9]$ & $84 \pm 16$ \\
Red Nuc. & $86 \pm 14[12]$ & $87 \pm 13[11]$ & $86 \pm 16[17]$ & $191 \pm 17[17]$ & $189 \pm 17[16]$ & $189 \pm 26$ \\
Subs. Nigra & $161 \pm 23[21]$ & $163 \pm 21[17]$ & $162 \pm 20[17]$ & $87 \pm 12[11]$ & $88 \pm 12[12]$ & $90 \pm 16$ \\
Dent. Nuc. & $103 \pm 12[19]$ & $112 \pm 12[13]$ & $108 \pm 12[15]$ & $110 \pm 11[13]$ & $164 \pm 21[16]$ & $174 \pm 24$ \\
Genu (CC) & $-19 \pm 2[5]$ & $-20 \pm 3[5]$ & $-19 \pm 3[5]$ & $-19 \pm 2[5]$ & $-18 \pm 3[6]$ & $-22 \pm 4$ \\
Splenium (CC) & $-18 \pm 5[3]$ & $-15 \pm 9[7]$ & $-15 \pm 9[6]$ & $-15 \pm 8[6]$ & $-15 \pm 8[6]$ & $-17 \pm 5$ \\
Capsula Int. & $-45 \pm 8[10]$ & $-51 \pm 9[13]$ & $-49 \pm 9[12]$ & $-46 \pm 8[10]$ & $-48 \pm 9[11]$ & $-43 \pm 8$ \\
Frontal WM & $-13 \pm 3[4]$ & $-17 \pm 6[7]$ & $-17 \pm 5[6]$ & $-15 \pm 4[5]$ & $-14 \pm 5[6]$ & $-16 \pm 5$ \\
Occ WM & $-31 \pm 4[10]$ & $-23 \pm 9[12]$ & $-27 \pm 7[8]$ & $-25 \pm 8[11]$ & $-28 \pm 7[8]$ & $-30 \pm 10$ \\
\hline GM & $116 \pm 55[15]$ & $119 \pm 53[13]$ & $117 \pm 54[13]$ & $119 \pm 53[13]$ & $117 \pm 54[13]$ & $120 \pm 55$ \\
WM & $-28 \pm 14[8]$ & $-29 \pm 17[10]$ & $-29 \pm 16[9]$ & $-27 \pm 15[8]$ & $-28 \pm 16[8]$ & $-28 \pm 13$ \\
GM+WM & $85 \pm 77[13]$ & $87 \pm 77[12]$ & $86 \pm 77[12]$ & $88 \pm 77[12]$ & $86 \pm 77[12]$ & $88 \pm 78$ \\
\hline
\end{tabular}

Abbreviations:

CC $=$ Corpus Callosus

$\mathrm{WM}=$ White Matter

$\mathrm{GM}=$ Gray Matter 
In vivo data. Results including the TGV-FANSI algorithm are shown in Figure S11 and S12.

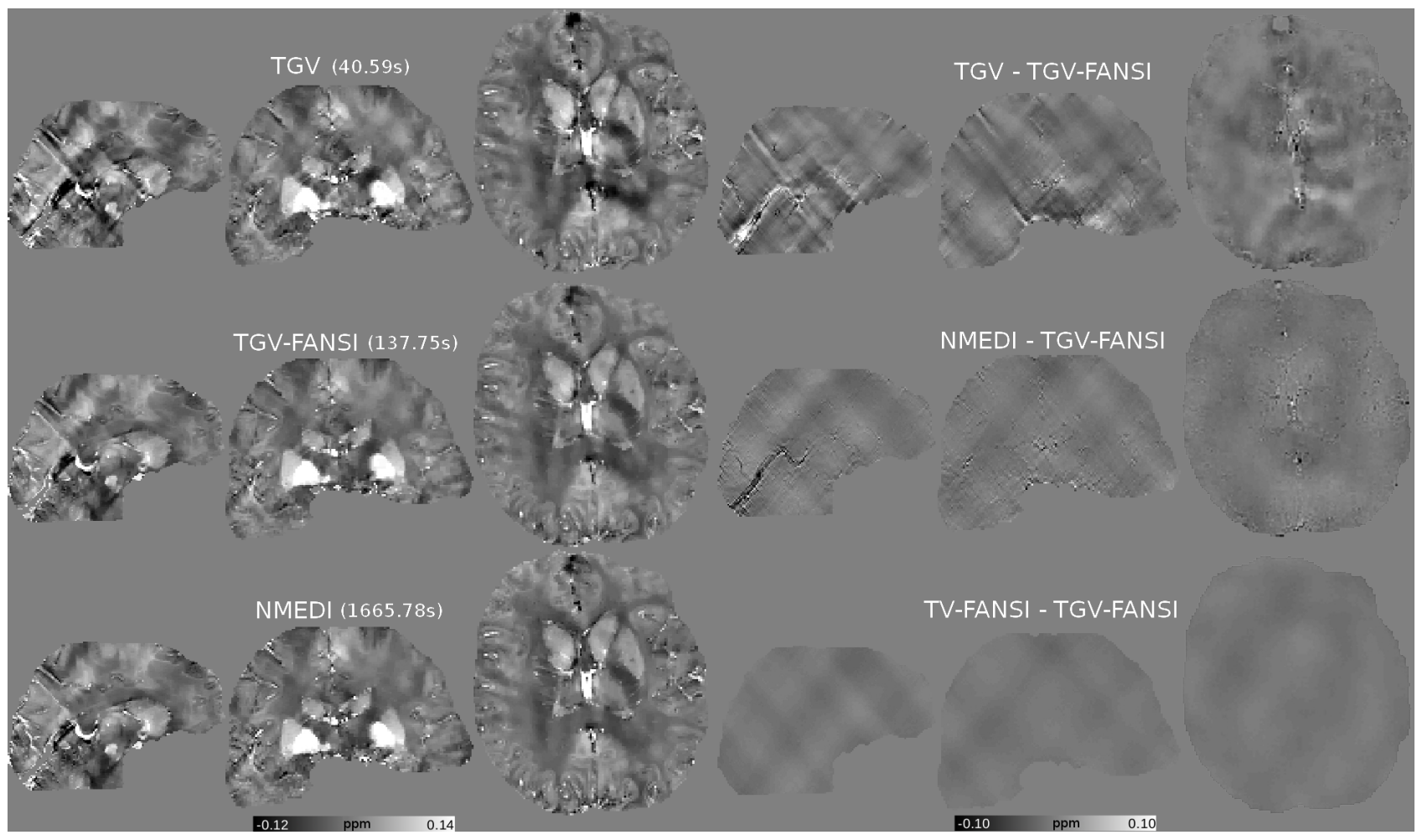

Fig. S11: Results for the 3T data and corresponding difference maps. Same ranges as Figure S12. 


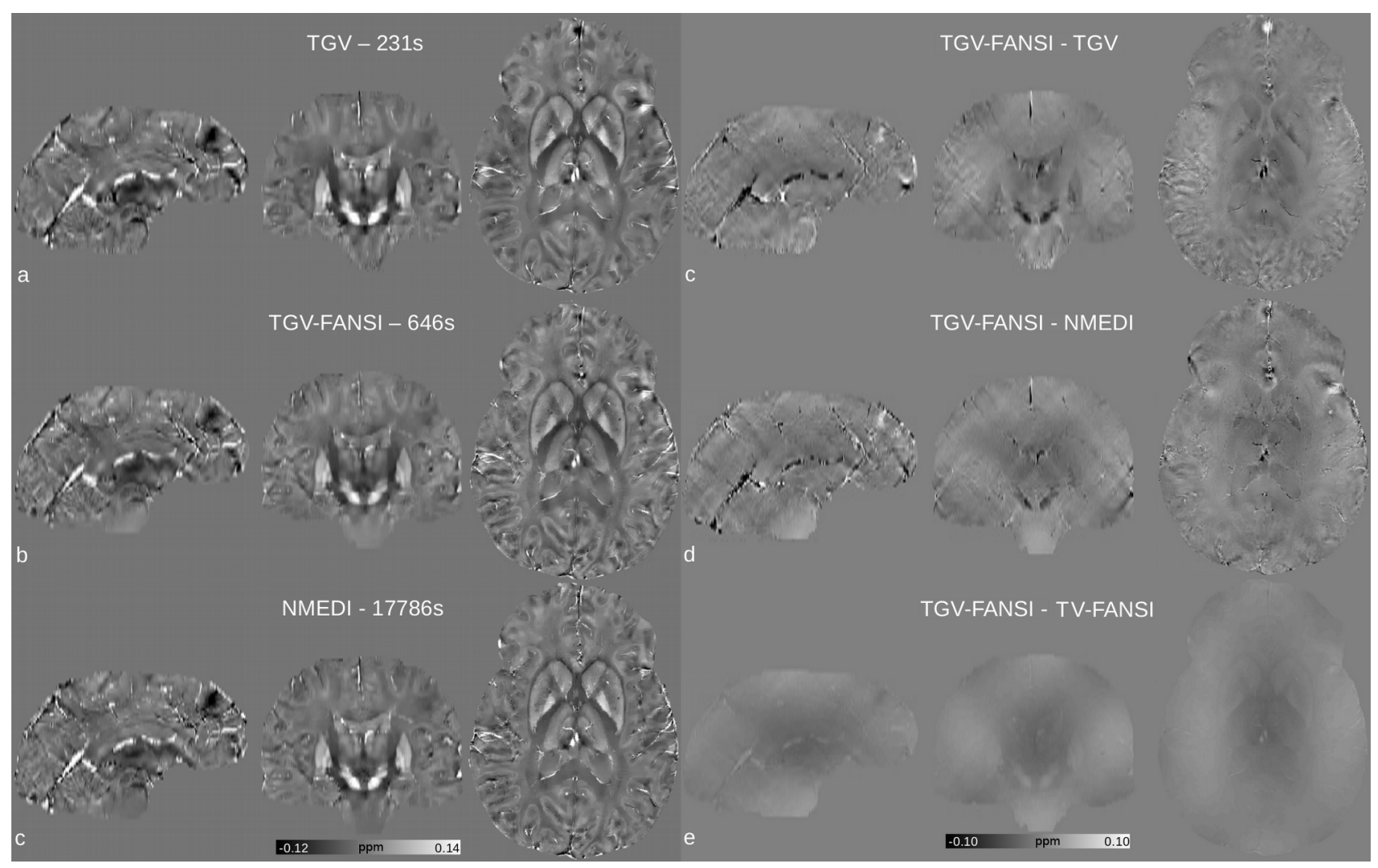

Fig. S12: Results for the 7T data and corresponding difference maps.

3T data: TGV-FANSI (running time: 138s) was 12.1 times faster than NMEDI.

7T data: TGV-FANSI (running time: 646s) was 27.5 times faster than NMEDI.

Regarding the choice of $\boldsymbol{\mu}_{1}$ and $\boldsymbol{\mu}_{0}$ parameters for the TGV regularization subproblems, as for TV, they were closely related to the actual regularization parameters, $\alpha_{0}$ and $\alpha_{1}$. It should be noted that although we set the relationship $\alpha_{0} / \alpha_{1}$ to 2 (as suggested in (13-17)), we found empirically that relationship $\mu_{0} / \mu_{1}=2$ also yielded acceptable results. This simplifies the problem to optimizing only $\alpha_{1}$ and $\mu_{1}$. As with the TV implementation, preliminary analyses suggest that optimal $\mu_{1} / \alpha_{1}$ might be restricted to the $[1,100]$ range.

TGV-FANSI was found to be 3 to 27 times faster than NMEDI and approximately 3 times slower than TV-FANSI. Thus, given both ADMM-based algorithms yielded similar results with the 
same $\alpha_{1}$ and $\mu_{1}$ parameters $\left(\alpha_{0} / \alpha_{1}\right.$ and $\mu_{0} / \mu_{1}$ within the $[0.5,2]$ range), we propose TV-FANSI could be used to fine-tune the regularization parameters - empirically via visual rating or using the L-curve method. Turning to algorithm performance, it is worth noting that all nonlinear algorithms yielded similar results, though with a small systematic advantage in quality metrics and accuracy for the newly proposed methods, for our synthetic experiments. Differences across nonlinear methods can be explained by the type of regularization employed; whilst NMEDI and TV-FANSI formulations promote piece-wise constant QSM reconstructions (in the case of NMEDI also aided by edge information in magnitude data - the impact of which can be gauged in Figure S5), TGV-FANSI promotes piece-wise smooth solutions. 